Editorial

\title{
Innovative Mobile Information Systems: Insights from Gulf Cooperation Countries and All Over the World
}

\author{
Miltiadis D. Lytras, ${ }^{1}$ Hassan Mathkour, ${ }^{2}$ and Miguel Torres-Ruiz ${ }^{3}$ \\ ${ }^{1}$ Computer Information Systems Department, The American College of Greece, Athens, Greece \\ ${ }^{2}$ King Saud University, Riyadh, Saudi Arabia \\ ${ }^{3}$ Instituto Politécnico Nacional, Mexico City, Mexico
}

Correspondence should be addressed to Miltiadis D. Lytras; mlytras@acg.edu

Received 17 July 2016; Accepted 29 August 2016

Copyright (C) 2016 Miltiadis D. Lytras et al. This is an open access article distributed under the Creative Commons Attribution License, which permits unrestricted use, distribution, and reproduction in any medium, provided the original work is properly cited.

\section{Introduction}

Mobile Information Systems and applications have received growing attention in recent years from various perspectives. The thriving numbers behind mobile systems adoption and contribution have captured the attention of computer engineering and business researchers that, in the past years, have been trying to decipher the phenomenon of mobile information systems, its relation to already-conducted research, and its implications for new research opportunities that effect innovation, entrepreneurship, and world economy dynamics.

The current mobile information systems and applications show cases in the Gulf Cooperation Council countries landscape and worldwide presenting a very interesting picture. Several big scale information systems provide a variety of services to local citizens and foreigners promoting top quality research towards the enhancement of the local economies and their transformation to knowledge based high effective sustainable economies. These widely accepted mobile information systems endeavors demonstrate that a wide range of mobile applications are available and present a viable and robust alternative to traditional desktop stand-alone solutions.

The objective of the special issue is to communicate and disseminate recent computer engineering and business research and success stories that demonstrate the power of mobile computing to improve traditional information technologies and their exploitation approaches. The purpose of the special issue is to demonstrate state-of-the-art approaches of mobile information systems that have had successful application and to show how new and advanced business models and adoption strategies can expand the sustainability frontiers in advanced applied computer engineering and update the global agenda of innovations in mobile information systems and applications.

\section{The Enabling Technologies for Innovative Mobile Information Systems through the Meta-Analysis of Contributions in the Special Issue}

The mobile information systems will continue globally to serve as enablers of innovation and will continue to promote entrepreneurship in sustainable ways. In this section, we are elaborating on the main contributions of the special issue as well as on future research directions and hot areas of research related to this domain. We are providing our basic conclusions and recommendations for future innovative mobile information systems agenda in relevance to five enabling technologies and four areas of innovation (see Figure 1). We also link the main contributions in this special issue related to these important areas:

\section{(i) Internet of Things}

Automated Bidirectional Languages Localization Testing for Android Apps with Rich GUI, A. M. A. Awwad and W. Slany 


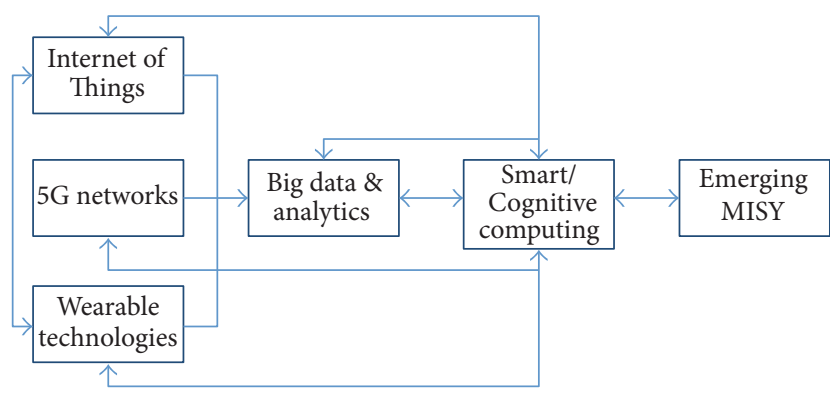

FIGURE 1: The enabling technologies for innovative mobile information systems.

Mobile Based User-Centered Learning Environment for Adult Absolute Illiterates, I. ur-Rehman et al.

\section{(ii) $5 G$ Networks}

Innovative Mobile E-Healthcare Systems: A New Rule-Based Cache Replacement Strategy Using Least Profit Values, R. A. Haraty

Towards Mobile Information Systems for Indoor Space, X. Zhang et al.

\section{(iii) Wearable Technologies/Virtual Reality}

Development of a Lunar-Phase Observation System Based on Augmented Reality and Mobile Learning Technologies, W. Tarng et al.

From Mobile to Wearable System: A Wearable RFID System to Enhance Teaching and Learning Conditions, S. L. Marie-Sainte et al.

\section{(iv) Big Data and Analytics}

Toward a Nationwide Mobile-Based Public Healthcare Service System with Wireless Sensor Networks, C. Shen et al.

Modeling Learners' Readiness to Adopt Mobile Learning: A Perspective from a GCC Higher Education Institution, M. Shorfuzzaman and M. Alhussein

Trace Attack against Biometric Mobile Applications, S. Ghouzali et al.

\section{(v) Smart/Cognitive Computing}

Quality Evaluation of Mobile Game: Miftah Alfasaha, G. Alhuhud and W. Altamimi

Classification of Arabic Twitter Users: A Study Based on User Behaviour and Interests, A. Alabdullatif et al.

A Mobile Information System Based on CrowdSensed and Official Crime Data for Finding Safe Routes: A Case Study of Mexico City, F. Mata et al.

With the integration of Internet of Things research, the perceived areas of application of mobile computing will be expanded further. $5 \mathrm{G}$ networks and new innovative approaches in networking protocols and standards will set up amazing capabilities for the provision of ubiquitous and pervasive services. Wearable and haptic technologies revolutionize also the borders of mobile information systems and permit new amazing behavioral and cognitive based perceptions for the exploitation of information and the integration of providers and consumers of Smart Data. The advanced capabilities of data analytics of our times and big data set new challenges for intelligent smart components accessible through mobile information systems. Moreover, advanced considerations on Machine Learning, Open Artificial Intelligence, and Cloud Computing infrastructures provide more complicated context for exploitation in academia, business, and industry.

\section{The Open Research Issues for Innovative Mobile Information Systems}

As a conclusion to this special issue, we want to communicate a reference model for next generation innovative mobile information systems and services linked to three critical design variables namely Content, Context, and Cognition.

Content refers to the dynamic collaborative constructed information with meaningful enhancements from mobile intelligent agents, which have the capacity to manage big data in micro and global contexts. A revolution to the content perception is that most of the applications will act as facilitators of content generated by users and the valuable contribution of MISY would be to provide value reference models to information.

Context refers to the mode of exploitation and the metalevel of reference that explores the capacity of MISY to personalize content and to initiate dialogic explorations of value through services.

Cognition is the new impressive dimension of next generation MISY. To our understanding, the integration of Deep Learning and Open Artificial Intelligence accompanied with Semantic Web ontological engineering methods and practices will strengthen the capacity of MISY to respond as problem solvers to real world cases. This convergence to our opinion serves as the new significant milestone for the evolution of mobile information systems research. There are several case studies to consider almost in every aspect of human life.

In Figure 2, we provide a classification model of innovative mobile information systems though the integration of Context and Cognition aspects. This can be useful for students interested in pursuing a Ph.D. degree or industry experts interested in realizing the potential of real world services.

The overall idea is that scalable solutions at micro, wide, and global level will generate an unforeseen, ubiquitous, and pervasive innovative mobile services infrastructure. For any of these twenty-two indicative types of innovative mobile services and nine clusters of potential challenging business models, there is a lot of research to be done and a number of interdisciplinary contributions are required. Few interesting case studies are discussed below with reference to human activity areas. 

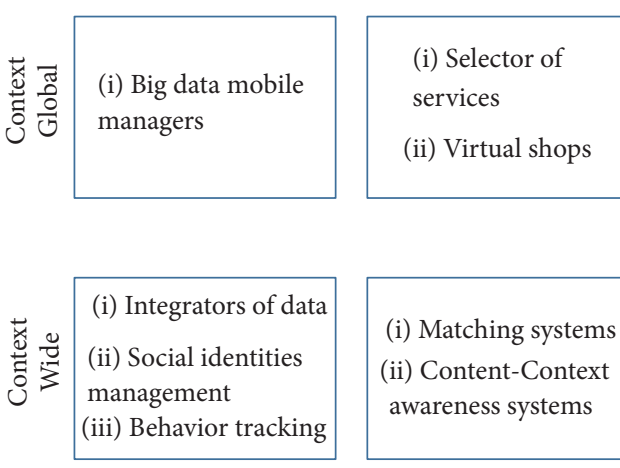

(i) Selector of services

(ii) Virtual shops awareness systems (i) Megatrends

managers

(ii) Global open AI

solutions

(iii) Robotics

(i) Science assistants

(ii) Intelligent agents

at wide scale

(iii) Problem solvers

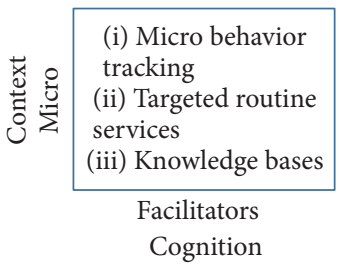
(i) Tracking systems
(ii) Behavior and cognition based recommenders
Recommenders
Cognition
(i) Intelligent agents
(ii) Domain specific bots
(iii) Human life
assistants
Smart Apps
Cognition

FIgURE 2: Context and Cognition as reference models for MISY.

Facilitators of Cognition/Microcontext. A very interesting category of next generation innovative mobile services would be related to microbehavior tracking. There are several examples associated with this: Consider applications capable of analyzing through sentiment analysis the feelings or mood of people in social networks. For example, psychotherapeutic services for people feeling lonely or isolated would be a good case. Another example from the Health Domain is the use of wearable technologies and tracking or sensor systems in order to collect critical information related to key indicators of biological life. Ph.D. studies on these specific areas can be diversified and focused on various disciplines:

(i) Semantic annotation schemas for microbehavior

(ii) Standardization of services for microbehavior

(iii) Cognitive systems for mental assistance

(iv) Psychotherapeutic assistants

Smart Applications/Microcontext. The integration of Deep Learning or advanced considerations of Artificial Intelligence combined with wearable and haptic technologies can easily justify applications and business innovations related to Human Life Assistants. These intelligent MISY can provide assistance related to medical treatment, cognitive monitoring, and training. Several Ph.D. studies could focus on these areas as well:

(i) Deep Learning algorithms for Human Activity Assistance

(ii) Open AI architectures for elderly assistance

The previous discussion can be seen in more complicated visioning scenarios. Consider the integration of Content, Context, and Cognition in a $3 \mathrm{D}$ dimensional space where the intersection of the three areas provides imaginary scenarios for next generation innovative mobile services. If we add more complexity in this space like problem solving or decision type or innovation type then the resulting framework is impressive. We are planning to publish a book for this discussion in 2017.

Another important dimension of the analysis for the future innovative mobile information systems is their linkage to well defined business models and sustainable innovation. There is a global discussion about new startups, cloud businesses, virtual enterprises, or businesses as ubiquitous services. In Table 1, we provide a conceptual reference model for the convergence of the emerging technologies towards sustainable innovation and emerging business models. We are encouraging researchers to deal with open issues related to this reference model and to investigate the value diffusion of technologies, to services, businesses, and economy. Technology sometimes should be considered as the black box of innovation. Creative thinking and creative entrepreneurship should construct new ideas for services that go further the perceptions about state-of-the-art businesses. Only by doing so, technology integrations can result in sustainable businesses. There is a worldwide concern for the capacity of new technologies to support economic efficacy and development. The abstraction of Table 1 is an interesting theoretical construct that permits different interpretation from technology or business.

\section{Discussion and Conclusions for the Contribution of the Special Issue to Innovative Mobile Information Systems}

We are moving fast towards a global ubiquitous and pervasive infrastructure of value adding services. At the same moment, 
TABLE 1: Innovation Clusters and Business Models for next generation mobile information systems.

\begin{tabular}{|c|c|c|}
\hline \multirow{2}{*}{ Technologies } & \multicolumn{2}{|c|}{ Next generation mobile information systems research } \\
\hline & Innovation cluster & Business models \\
\hline $\begin{array}{l}\text { Mobile networks/networking } \\
\text { technologies/sensor networks }\end{array}$ & $\begin{array}{l}\text { (i) Tracking systems } \\
\text { (ii) Smart content sharing } \\
\text { (iii) Antipoverty networks } \\
\text { (iv) Personalized services in health, education } \\
\text { (v) Medical mobile systems innovations } \\
\text { (vi) Urban computing applications at micro and } \\
\text { wide levels } \\
\text { (vii) Protocols and standardization } \\
\text { (viii) Traffic control } \\
\text { (ix) Security performance and control }\end{array}$ & $\begin{array}{l}\text { (i) Community building } \\
\text { (ii) Micronetworking } \\
\text { (iii) MISY as services } \\
\text { (iv) MISY as infrastructure }\end{array}$ \\
\hline Cognitive computing & $\begin{array}{l}\text { (i) Prediction systems } \\
\text { (ii) Services selection } \\
\text { (iii) Management of behavior and cognition } \\
\text { (iv) Large scale mobile content integration }\end{array}$ & $\begin{array}{l}\text { (i) Mobile profiling in microapplications } \\
\text { (ii) Collective wisdom projects } \\
\text { (iii) Human networking analysis } \\
\text { (iv) Behavior control }\end{array}$ \\
\hline Big data and business analytics & $\begin{array}{l}\text { (i) Patterns recognition for linked data in MISY } \\
\text { (ii) Smart cities services } \\
\text { (iii) Deep Learning and neural networks for } \\
\text { services allocation and prediction } \\
\text { (iv) Semantic queries and reasoning } \\
\text { (v) Financial advisors }\end{array}$ & $\begin{array}{l}\text { (i) Social innovation } \\
\text { (ii) Smart communities } \\
\text { (iii) Dynamic composition of MISY platforms } \\
\text { (iv) Distributed networks integration } \\
\text { (v) Human activities as services } \\
\text { (vi) MISY driven business analytics }\end{array}$ \\
\hline Internet of Things & $\begin{array}{l}\text { (i) Marketplaces of everything } \\
\text { (ii) Urban Growth, value adding urban services } \\
\text { (iii) Annotation of services and applications } \\
\text { (iv) Rating systems } \\
\text { (v) Wearable innovation }\end{array}$ & $\begin{array}{l}\text { (i) Systematic exploration of profiles and groups } \\
\text { (ii) Marketplaces of everything } \\
\text { (iii) Economic development networks } \\
\text { (iv) Cross-cultural skills transfer } \\
\text { (v) Wisdom management }\end{array}$ \\
\hline $\begin{array}{l}\text { Human computer interaction, } \\
\text { haptic technologies, and virtual } \\
\text { reality }\end{array}$ & $\begin{array}{l}\text { (i) Haptic technologies for disabled } \\
\text { (ii) Therapeutic MISY for health domain } \\
\text { (iii) Voice recognition in MISY } \\
\text { (iv) Navigation and tracking } \\
\text { (v) Smart visualization systems }\end{array}$ & $\begin{array}{l}\text { (i) Advanced visualization networks for people, } \\
\text { community, and services } \\
\text { (ii) Personal identities management and the } \\
\text { virtual self } \\
\text { (iii) Immersive reality }\end{array}$ \\
\hline
\end{tabular}

we are realizing a revolution on micro-local-aware services with the exploitation of sensor networks and tracking systems. The following are a few conclusions derived from metaanalysis of the research papers of this special issue and our personal dispositions and thoughts:

(i) The convergence of a variety of technologies will define the new value proposition for innovative mobile information systems and will set up a new perspective on the basic nature of mobile information systems. We consider Internet of Things technologies, Cognitive computing, Smart Data, and Immersive Virtual Reality as the catalysts of change.

(ii) Cross-domain considerations will define brand new markets for services and real world innovative mobile information systems. Diverse scientific areas and domains of human activity provide challenging business cases. For example, innovative mobile information systems applications for Health Domain and Analytics based on Cognition, Psychotherapeutic systems and Immersive innovative mobile information systems, IoT, and Smart Data services.

(iii) Innovative mobile information systems research related to business and innovation issues will have the same significance as research on the technical aspects. To our perception, there is a current critical gap in business considerations.

(iv) The Innovation linked to mobile information systems should investigate new value proposition models. The evolution of last decade has set innovative mobile information systems research area as a mature scientific domain. It is time to enrich the perceptions with more sophisticated models of value diffusion.

(v) There is still plenty of space for robust mobile services related to very simple ideas and concepts. It is a challenge for scientists and entrepreneurs to promote cross-cultural collaboration. We do believe that most of these sustainable economic services will exactly focus on the exploitation of cross-cultural understanding and joint effort.

(vi) The convergence of innovative mobile information systems with IoT will set all the prerequisites for the development of a related science like Web Science or Data Science.

Our objective in order to exploit the synergies of this special issue is to publish in 2017 a new special issue on Innovation 
Clusters and Sustainable Business Models for innovative mobile information systems.

Miltiadis D. Lytras

Hassan Mathkour

Miguel Torres-Ruiz 

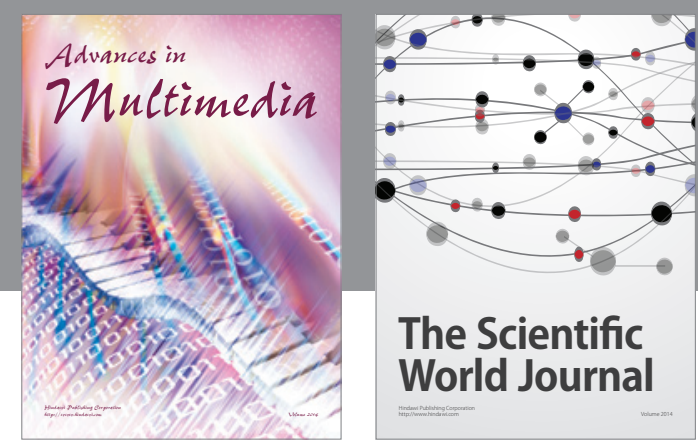

The Scientific World Journal
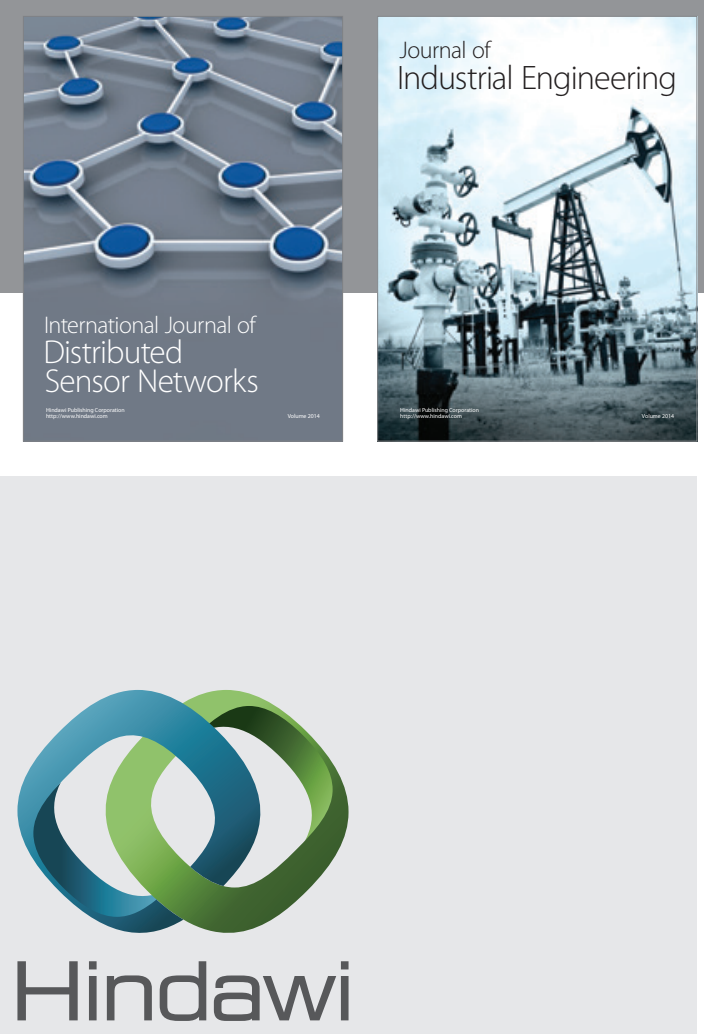

Submit your manuscripts at

http://www.hindawi.com

\section{Computer Networks} and Communications
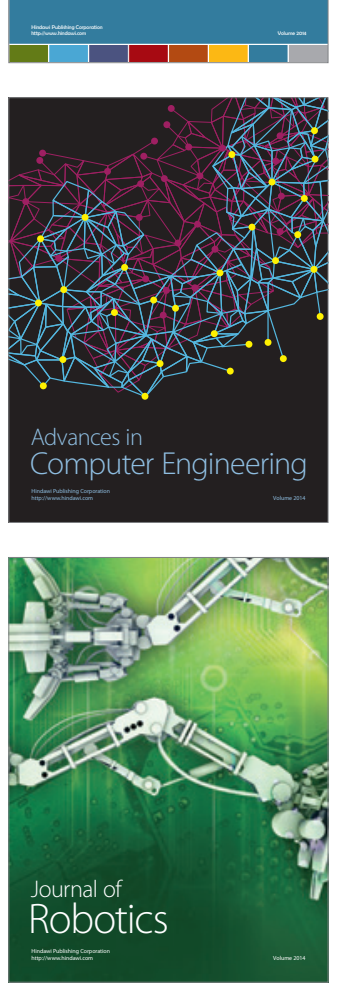
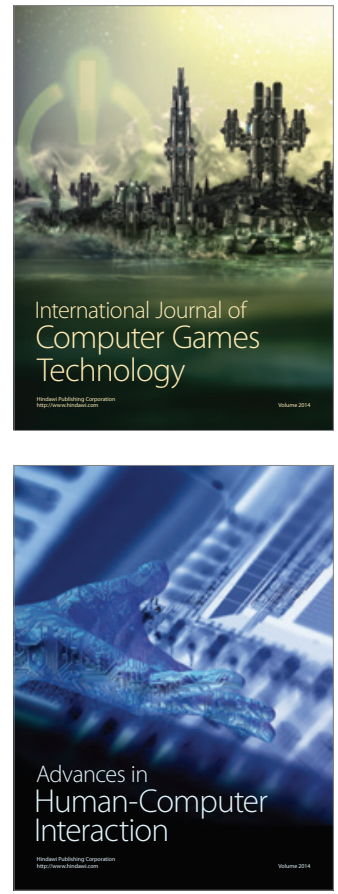
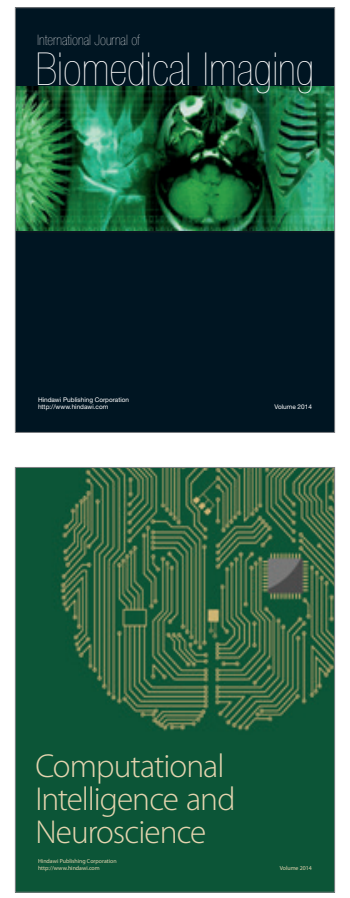
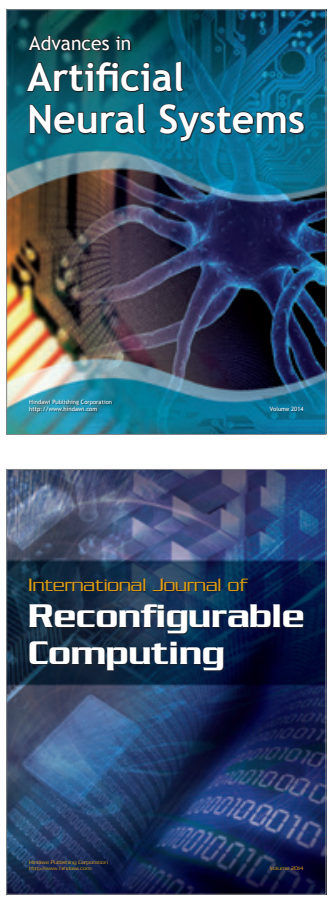
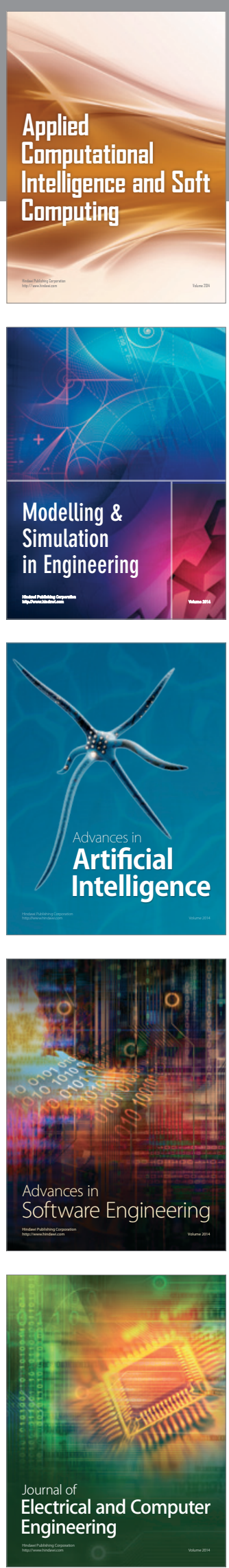